Vietnam Journal of Mechanics, VAST, Vol.31, No. 2 (2009), pp. 103-121

\title{
COMBINED MOTION OF RESERVOIR WITH LIQUID FOR ANGULAR MOTIONS OF CARRYING BODY
}

\author{
Oleg S. Limarchenko \\ The Taras Shevchenko Kiev National University, Ukraine
}

\begin{abstract}
We consider peculiarities of the modeling of wave motion of ideal liquid in a reservoir, which performs inclined motions. For description of behavior of the system we use variational formulation of the problem on the basis of the Hamilton-Ostrogradskiy variational principle with preliminary satisfying all kinematic boundary conditions of the problem. It is shown that this approach makes it possible to reduce considerably the number of unknowns of the problem and reduce it description only to parameters of motion of a liquid free surface and parameters of motion of a carrying body. The constructed nonlinear discrete model of the system was applied for investigation of motion of a reservoir with liquid on pendulum suspension, on taking into account liquid outflow and other problems, which have theoretical and applied meaning.
\end{abstract}

\section{INTRODUCTION}

It is known that problems about combined motion of reservoirs with liquid represent significant part of investigations in the field of wave motions of liquid, which have great theoretical and applied significance. Theoretically this problem is especially complicated and important in the case, when liquid partially fills empty of a rigid body. Investigations of such types of problems by theoretical and experimental methods are performed during several centuries. It is interesting that Relay, Faraday and Ostrogradskiy $[2,14$, 16] were pioneers in investigation of such problems. Special theoretical and applied interest to these problems appeared because of development of rocket engineering. Investigations of 50-s made it possible to develop linear and nonlinear theories of motion of such systems. Mainly the case of given translational motion of carrying body was under investigation. However, in the case when carrying body performs angular (inclined) motions the number of publications is very small $[1,3,5,8,15,17,18]$, especially in the case when combined motion of carrying body and liquid with a free surface is investigated. In publications of N.E. Zhukovskiy [5] was created fundamentals of the theory of motion of rigid bodies with empties completely filled by ideal incompressible liquid, which performs irrotational motion. Attempts to generalize these results in the case of motion of liquid with a free surface result in very awkward and hard for investigation mathematical problems. The problem becomes more complicated in the case of nonlinear statement. Importance of such problems in applied aspect is caused by the fact that in the case of great relative mass of liquid its wave motion can influence considerably on dynamics of transport vehicle (carrying body). 
In publications of V. V. Roumyantsev, G. S. Narimanov, I. A. Lukovskiy, L. V. Dokuchaev $[9,12,13]$ motion equations for nonlinear model of reservoirs with ideal liquid with a free surface in the case, when carrying body performs not only translational but rotational motion, were obtained. At the same time realization of these algorithms on numerical examples until now was not performed. Complexities of such numerical realization are conditioned by the property that on angular motions of the carrying body it is necessary to introduce into consideration supplementary vector potential of velocities $\vec{\Omega}$ (i.e., three scalar potentials) along with before introduced one scalar potential of velocities $\varphi$. Therewith expressions for this vector potential are obtained more complicated than for $\varphi$. Total volume of calculations increases considerably.

In the present article we suggest to realize modeling of dynamics of the system body-liquid with a free surface on the basis of the method, which uses the HamiltonOstrigradskiy variational principle with preliminary analytical elimination of all kinematic boundary conditions of the problem. Fundamentals of this method were stated in publications $[7,8]$. Main attention is focused on questions of obtaining the model of minimal dimensionality and its application to a number of applied problems, in which interaction of angular motions of the carrying body and wave motion of liquid is determinative.

\section{OBJECT OF INVESTIGATION AND MATHEMATICAL STATEMENT OF THE PROBLEM}

Let us consider a cylindrical reservoir of arbitrary cross-section with absolutely rigid walls, which is partially filled by ideal homogeneous liquid subjected to capillary forces.

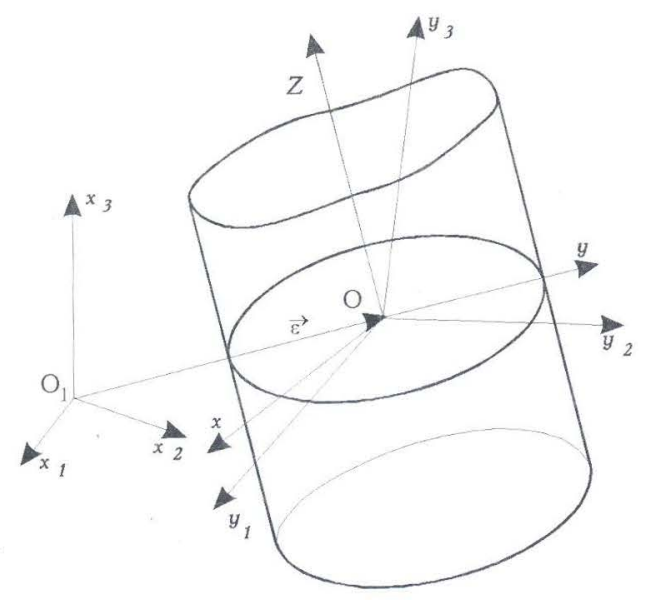

Fig. 1. Reference frames

We introduce conventionally immovable reference frame $O_{1} X_{1} X_{2} X_{3}$, the reference frame $O x y z$, fixed with reservoir and the system $O Y_{1} Y_{2} Y_{3}$ with the origin at the point $O$, which axes are correspondingly parallel to axes of the system $O_{1} X_{1} X_{2} X_{3}$ (Figure 1). The point $O$ is selected at certain internal point of initially unperturbed free surface of 
liquid (at the center of unperturbed free surface of liquid, if it exists), and the axis $O z$ is directed towards external normal to unperturbed free surface. Motion of the point $O$ in the reference frame $O_{1} X_{1} X_{2} X_{3}$ is given by the radius-vector $\vec{\varepsilon}(t)$, and rotational motion of the system $O x y z$ relative to $O_{1} X_{1} X_{2} X_{3}$ is given by three turn angles $\alpha_{1}, \alpha_{2}, \alpha_{3}$. Here we define the angle $\alpha_{1}$ as turn angle of the system $O x y z$ relative to the axis $O Y_{1}$, the angle $\alpha_{2}$ corresponds to rotation of the system relative te new position of the axis $O Y_{2}$, and $\alpha_{3}$ corresponds to rotation of the system relative to new position of the axis $O Y_{3}$. Let us introduce into consideration unit vectors of the reference frame $O Y_{1} Y_{2} Y_{3}$, i.e., $\left\{\vec{y}_{1}^{0}, \vec{y}_{2}^{0}, \vec{y}_{3}^{0}\right\}$ and for the reference frame Oxyz, i.e., $\left\{\vec{x}^{0}, \vec{y}^{0}, \vec{z}^{0}\right\}=\left\{\vec{i}_{1}, \vec{i}_{2}, \vec{i}_{3}\right\}$.

Then, it is possible to represent the transition matrix as

$$
\begin{aligned}
& \vec{y}_{i}^{0}=e_{i j} \vec{i}_{j}, \quad \text { where } e_{11}=\cos \alpha_{2} \cos \alpha_{3} ; e_{12}=-\cos \alpha_{2} \sin \alpha_{3} ; \\
& e_{13}=\cos \alpha_{1} ; e_{21}=\cos \alpha_{1} \sin \alpha_{3}+\sin \alpha_{1} \sin \alpha_{2} \cos \alpha_{3} ; \\
& e_{22}=\cos \alpha_{1} \cos \alpha_{3}-\sin \alpha_{1} \sin \alpha_{2} \sin \alpha_{3} ; e_{23}=-\sin \alpha_{1} \cos \alpha_{2} ; \\
& e_{31}=\sin \alpha_{1} \sin \alpha_{3}-\cos \alpha_{1} \sin \alpha_{2} \cos \alpha_{3} ; \\
& e_{32}=\sin \alpha_{1} \cos \alpha_{3}-\cos \alpha_{1} \sin \alpha_{2} \sin \alpha_{3} ; e_{33}=\cos \alpha_{1} \cos \alpha_{2} .
\end{aligned}
$$

Expressions for components of angular velocity $\vec{\omega}$ in the fixed reference frame will be (dot above variables denotes differentiation by time $t$ )

$$
\begin{aligned}
& \omega_{x}=\omega_{1}=\dot{\alpha}_{1} \cos \alpha_{2} \cos \alpha_{3}+\dot{\alpha}_{2} \sin \alpha_{3} ; \\
& \omega_{y}=\omega_{2}=-\dot{\alpha}_{1} \cos \alpha_{2} \sin \alpha_{3}+\dot{\alpha}_{3} \cos \alpha_{3} ; \\
& \omega_{z}=\omega_{3}=\dot{\alpha}_{1} \sin \alpha_{2}+\dot{\alpha}_{3} .
\end{aligned}
$$

Thus, the system of parameters $\varepsilon_{i}$ and $\alpha_{i}$ with taking into account introduced angles (1), (2) completely characterizes motion of reservoirs in conventionally immovable reference frame $O_{1} X_{1} X_{2} X_{3}$.

For description of motion of limited volume of liquid we introduce the following denotations. Let $\tau$ be the domain occupied by liquid, $S$ be the free surface of liquid, $\Sigma$ be the moisten surface of liquid, $L$ be the contour of contact of three media gas-body-liquid. Here the absence of index in these denotations indicates that these denotations are used for perturbed volume, and the presence of the index "zero" corresponds to unperturbed volume of liquid.

We assume that liquid at initial time instant was vortex-free, then taking into account its ideality and homogeneity for all following time instants motion of liquid will be potential. So, we introduce the function of velocity potential $\Phi(\vec{r}, t)$. Then, absolute $\vec{v}_{a}$ and relative $\vec{v}_{r}$ velocities of motion of liquid particles will be

$$
\begin{aligned}
& \vec{v}_{a}=\vec{\nabla} \Phi ; \\
& \vec{v}_{r}=\vec{\nabla} \Phi-\vec{\omega} \cdot \vec{r}-\dot{\vec{\varepsilon}}
\end{aligned}
$$

Due to specificity of the considered problem, when the domain of liquid has a part of boundary, which is free and beforehand unknown, we introduce into consideration the equation of $\stackrel{2}{\mathrm{~A}}$ free surface

$$
z=\xi(x, y, t) \text { or } z=\xi(r, \theta, t)
$$


Potential of resolving of the equation of a free surface relative to $z$ is predetermined by cylindric shape of empty of the rigid body. Here the first equation in (5) corresponds to the case of rectangular Cartesian coordinate system, and the second one corresponds to cylindrical coordinate system. The equation $z=0$ defines unperturbed free surface of liquid $S_{0}$.

According to publications [5, 13], for solving the mentioned class of problems it is convenient to represent the velocity potential $\Phi$ as

$$
\Phi=\varphi_{0}+\dot{\vec{\varepsilon}} \cdot \vec{r}+\vec{\omega} \cdot \vec{\Omega},
$$

here $\varphi=\varphi_{0}+\dot{\vec{\varepsilon}} \cdot \vec{r}, \vec{r}$ is radius-vector of arbitrary point in fixed reference frame. Actually in the expression for the velocity potential (6) the first addend corresponds namely to wave motion, the second one corresponds to connection of wave motion of liquid with translational motion of the reservoir, and the third one corresponds to connection of wave motion of liquid with rotational motion of the reservoir. It is seen from the relation (6), that on investigation of rotational motion of the carrying body three new unknown scalar functions, i.e., components of $\vec{\Omega}$ are introduced into consideration, for which in addition boundary value problems are formulated in more complicate form that for scalar component of the velocity potential $\varphi$.

Mathematical formulation of the problem about motion of the system reservoir liquid with a free surface can be reduced to a system of dependencies, which represents requirements of kinematic character, dynamic equations and initial conditions. From the point of view of general properties of description of mechanical systems on the basis of the Hamilton-Ostrogradskiy principle it is necessary to consider kinematic conditions as mechanic constraints, which superimpose restrictions on variations of unknowns. Dynamical boundary conditions here are obtained from the Hamilton-Ostrogradskiy principle as natural ones. For the investigated mechanical system we should consider kinematical requirements about: the condition of continuity of liquid flow in the domain $\tau$

$$
\Delta \varphi=0 ; \Delta \vec{\Omega}=0 \text { in } \tau,
$$

nonflowing conditions on the boundary of contact body - liquid $\Sigma$ with unit outer normal $\vec{n}$

$$
\frac{\partial \varphi_{0}}{\partial n}=0 \quad \text { on } \quad \Sigma ; \quad \frac{\partial \Omega}{\partial n}=\vec{r} \cdot \vec{n} \quad \Sigma+S_{0} ;
$$

and nonflowing conditions through a free surface of liquid, obtained with taking into account (3), (4)

$$
\begin{aligned}
& \frac{\partial \xi}{\partial t}+\vec{\nabla} \xi \cdot\left[\vec{\nabla} \varphi_{0}+\vec{\nabla}(\vec{\omega} \cdot \vec{\Omega})-\dot{\vec{\varepsilon}}-\vec{\omega} \times \vec{r}\right] \\
& =\frac{\partial \varphi_{0}}{\partial z}+\vec{\omega} \cdot \frac{\partial \vec{\Omega}}{\partial z}-\dot{\varepsilon}_{z}-\left.(\vec{\omega} \times \vec{r})\right|_{z} \text { on } S_{\xi}
\end{aligned}
$$

Dynamic boundary conditions and equations of motion of the reservoir can be obtained on the basis of the Hamilton-Ostrogradskiy variational principle with the Lagrange 
function

$$
\begin{aligned}
\mathcal{L}= & \frac{1}{2} \rho \int_{\tau}[\vec{\nabla} \varphi+\vec{\nabla}(\vec{\omega} \cdot \vec{\Omega})]^{2} d \tau+\frac{1}{2} M_{r}(\dot{\vec{\varepsilon}})^{2}+\frac{1}{2} I_{\text {res }}^{i j} \omega_{i} \omega_{j} \\
& -\left(M_{r}+M_{1}\right) g \varepsilon_{z}+\rho g\left(\cos \alpha_{1} \sin \alpha_{2} \cos \alpha_{3}-\sin \alpha_{1} \sin \alpha_{3}\right) \\
& \times \int_{S_{0}} r \cos \theta(\xi+H) d S-\rho g\left(\sin \alpha_{1} \cos \alpha_{3}+\cos \alpha_{1} \sin \alpha_{2} \sin \alpha_{3}\right) \\
& \times \int_{S_{0}} r \sin \theta(\xi+H) d S-\frac{1}{2} \rho g \cos \alpha_{1} \cos \alpha_{2} \int_{S_{0}} \xi^{2} d S \\
& -\left(M_{1} h_{1}+M_{r} h_{r}\right)\left(1-\cos \alpha_{1} \cos \alpha_{2}\right)-\sigma \int_{S_{0}} \sqrt{1+(\vec{\nabla} \xi)^{2}} d S \\
& -\sigma \cos \theta_{1} \int_{L_{0}} \xi d l+\vec{F} \cdot \vec{\varepsilon}+\vec{M} \cdot \vec{\chi} .
\end{aligned}
$$

In the relation (10) we used the following denotations: $\rho$ is liquid density, $g$ is free falling acceleration, $H$ is filling level, $M_{r}$ and $M_{1}$ are the mass of reservoir and liquid, $h_{1}$ and $h_{r}$ are displacements of mass centers of liquid and reservoir relative to the plane of unperturbed free surface of liquid $S_{0}, \sigma$ is surface tension on a free surface of liquid, $\theta_{1}$ is contact angle, $I_{\text {res }}^{i j}$ is inertia tensor of the reservoir, determined relative to the point $O$, $\vec{F}$ and $\vec{M}$ are main vector and main moment of external forces relative to the point $O$, which act on reservoir (representations potentials of external forces and moments are conventional), $\vec{\chi}=\left\{\alpha_{1}, \alpha_{2}, \alpha_{3}\right\}$ is conventional representation of turn angles of the reservoir relative to immovable reference frame.

The equations (7)-(9) relative to the variational principle

$$
\delta \int_{t_{1}}^{t_{2}} \mathcal{L} d t=0
$$

represent totality of kinematic constraints, which should be eliminated before solving the variational problem for efficient use of variational methods.

\section{CONSTRUCTION OF DECOMPOSITIONS OF DESIRED VARIABLES, WHICH HOLD ALL KINEMATIC CONSTRAINTS OF THE PROBLEM}

For efficient use of the Hamilton-Ostrogradskiy variational in principle it is necessary to construct representation of unknowns of the problem about motion of reservoir with liquid with a free surface, which in advance hold kinematic boundary conditions (7)-(9). According to publications $[6,8,11]$ we accept the following form to search solution of the 
problem about motion of liquid, which partially fills movable reservoir

$$
\begin{aligned}
\xi & =\sum_{n} a_{n}(t) \psi_{n}(x, y) ; \\
\varphi & =\sum_{n} b_{n}(t) \psi_{n}(x, y) \frac{\operatorname{ch} \varkappa_{n}(z+H)}{\varkappa_{n} \operatorname{sh} \varkappa_{n} H} ; \\
\vec{\Omega} & =\vec{\Omega}_{0}+\sum_{n} \vec{q}_{n}(t) \psi_{n}(x, y) \frac{\operatorname{ch} \varkappa_{n}(z+H)}{\varkappa_{n} \operatorname{sh} \varkappa_{n} H} .
\end{aligned}
$$

Here $\psi_{n}(x, y)$ is a complete orthogonal system of functions in the domain $S_{0}$, which can be found from the solution of the Neumann boundary value problem with the parameter $\varkappa_{n}$ :

$$
\Delta \psi_{k}+\varkappa_{k}^{2} \psi_{k}=0 \text { on } S_{0} ; \quad \frac{\partial \psi_{k}}{\partial n}=0 \text { on } L_{0}
$$

Here $L_{0}$ is a contour of contact of the unperturbed free surface of liquid $S_{0}$ with reservoir walls $\Sigma$. We note that the equation in the boundary value problem (15) is the consequence of the condition (7), while the boundary condition is the consequence of the relation (8). The vector-function $\vec{\Omega}_{0}$ represents the Stokes-Zhukovskiy potential, which is obtained as the solution of the Neumann problem for the Laplace equation

$$
\Delta \vec{\Omega}_{0}=0 ; \quad \frac{\partial \vec{\Omega}_{0}}{\partial n}=\vec{r} \times \vec{n} \text { on } S_{0}+\Sigma .
$$

This Neumann boundary problem was investigated in details in publications $[1,9,13,15]$, wherethe algorithms of solving for different shapes of empties were constructed.

The adduced representation of variables (12)-(14) in virtue of selection of the function $\psi_{k}$ holds identically the continuity equation (7) and nonflowing conditions (8). Decompositions (12)-(14) of variables $\xi, \varphi_{0}$ and $\vec{\Omega}_{0}$ coincides by form with solution of the problem about motion of bounded volume of liquid in movable reservoir obtaine within the framework of linear statement. So, actually according to the present approach we propose to search solution of nonlinear problem about combined motion of a reservoir with liquid in the form of decompositions by eigenfunctions of the linear problem.

The most complicated part in construction of independent (from the point of view of analytical mechanics) decompositions of variables $\xi, \varphi_{0}$ and $\vec{\Omega}_{0}$ is satisfying the kinematical boundary condition on a free surface of liquid (9). The problem of elimination of kinematic boundary condition on a free surface of liquid is typical for investigation of problems of dynamics of bodies with liquid and represents a component part of practically all existing methods used for solving problems of this class, especially methods based on use of velocity potential in analytical form. Such problem may be solved on the basis of the Fourier method [13], the Galerkin method [7, 8] and variational method [9, 18, 19] with application fundamentals of nonlinear mechanics. Here the problem about elimination of kinematic boundary condition on a free surface may be solved both independently of investigation of dynamical problem $[7,8,13,19]$ and as a component part of its solving in the case when kinematical boundary condition is natural for variational statement of the problem $[9$, 19] (use the variational statement of the problem based on the Bateman principle). Most 
efficiently this problem is solved in the case when coordinate functions are orthogonal and hold kinematical boundary conditions on moisten boundary. This fact indicates once more on expedience of usage of normal modes (obtained on the basis of solving of correspondent linear problems) as coordinate functions.

Let us consider the technique of elimination of kinematic boundary condition on a free surface, which is based on the application of the Galerkin method coupled with principles of nonlinear mechanics. In order to eliminate approximately kinematical boundary condition on a free surface of liquid it is necessary to select a set of basic parameters of the system, by which independent parameters will be further expressed. As it follows from theorem, vortex-free motion of ideal incompressible liquid is completely defined by motion of its boundaries, the number of degrees of freedom of the whole volume of liquid with a free surface is equal to the number of degrees of freedom of namely free surface. Hence, it is expedient to select the variable $\xi$ as the basic parameter of the system, which characterizes motion of liquid with a free surface, while variables $\varphi$ and $\Omega$ will be considered as dependent ones. Selection of independent parameters with application of decompositions (12)-(14) is realized in the following way: the system of amplitude parameters of decomposition of perturbations of a free surface into series by normal modes of liquid with a free surface is considered as independent, while parameters of decomposition into series of the scalar $\varphi$ and vector $\vec{\Omega}$ components of velocity potential $b_{i}$ and $\vec{q}_{i}$ correspondingly are considered as dependent on parameters $a_{i}$. Here the system of amplitude parameters $a_{i}$ together with parameters of motion of carrying body exhaustively characterizes kinematics of liquid with a free surface.

For determination of definite form of dependency of $b_{i}$ and $\vec{q}_{i}$ on $a_{k}$ we make use of the kinematical boundary condition on a free surface (9). Taking into account that kinematical boundary condition must hold for arbitrary laws of motion of reservoir, the condition (9) disintegrates into the following four conditions on a free surface $S$, i.e., for $z=\xi$

$$
\begin{aligned}
L^{(0)}\left(\xi, \varphi_{0}\right) & =\frac{\partial \xi}{\partial t}+\vec{\nabla} \xi \cdot \vec{\nabla} \varphi_{0}-\frac{\partial \varphi_{0}}{\partial z}=0 ; \\
L^{(k)}\left(\xi, \Omega_{0}\right) & =\vec{\nabla} \xi \cdot \vec{\nabla} \Omega_{k}-\frac{\partial \Omega_{k}}{\partial z}+\vec{i}_{k}\left(\vec{i}_{3} \times \vec{r}\right), \quad k=1,2,3 .
\end{aligned}
$$

Further we denote by $\Omega_{k}$ components of the vector component of velocity potential $\vec{\Omega}, L^{(i)}(f, g)$ are differential operators $(i=0,1,2,3)$. Thus, kinematical boundary condition from the point of view of mechanical parameters $a_{i}$ represents one nonholonomic (16) and three holonomic (17) mechanical constraints.

Let us give additional comments about potential of decomposition of the condition (9) into four conditions (16), (17), since obtaining four new conditions from one scalar condition looks unusual. On the one hand, as it was mentioned above, from the requirement of realization of the condition for arbitrary laws of reservoir motion (the property that the form of kinematic condition for liquid should not depend on the law of motion of both liquid and reservoir follows from definition of kinematical constraint) it follows that by considering in turn nonzero components $\vec{\omega}$, as well as the case when $\vec{\omega}=0$ we obtain four conditions for realization of the mentioned property of kinematical constraint. We 
can obtain the same result if we take into account that variations of variables, which characterize motion of liquid, and variables, which characterize motion of reservoir, are independent. Then if we vary kinematical boundary condition on a free surface, then with taking into account independence of of variations it disintegrates into several relations. Similar technique was used in publications of A.I. Lourie [10]. On the other hand, as it was shown above, in the considered case motion of liquid is completely defined by motion of its boundaries. Therefore, variables $\varphi$ and $\Omega_{k}$ depend only on one variable $\xi$. However, one relation (9) enables determination of only one dependence, therefore, three other dependencies may be selected arbitrary. It is expedient to select them from considerations of maximal simplification of the form of these dependencies. Namely the conditions (17) represent the form, which provides disintegration of conditions for $\Omega_{k}$ as independent ones.

Further procedure of determination of dependence of parameters $b_{i}$ and $\vec{q}_{i}$ on amplitude parameters $a_{i}$ is done on the basis of the Galerkin method. To this end we substitute decompositions (12)-(14) into differential operators $L^{(i)}(f, g)$, multiply by the system of functions $\psi_{k}$ and integrate over the domain $S_{0}$.

$$
\left.\int_{S_{0}} L^{(i)}\left(\xi, \begin{array}{c}
\varphi_{0} \\
\Omega_{k}
\end{array}\right)\right|_{S} \psi_{k} d S=0 \quad \begin{gathered}
i=0,1,2,3 \\
k=1,2, \ldots .
\end{gathered}
$$

Here immediate determination of values of differential operators $L^{(i)}$ on unknown free surface of liquid $S$, i.e., for $z=\xi$ is realized by means of projection of the given operator onto the surface $S_{0}$ with application of perturbation technique. Moreover, we shall look for dependence of values $b_{i}$ and $\vec{q}_{j}$ on $a_{k}$ as

$$
b_{i}=b_{i}^{(1)}+b_{i}^{(2)}+b_{i}^{(3)}+b_{i}^{(4)} ; \quad \vec{q}_{i}=\vec{q}_{i}^{(1)}+\vec{q}_{i}^{(2)}+\vec{q}_{i}^{(3)},
$$

where numerical indexes in brackets correspond to orders of smallness of values relative to $a_{i}$. As the result by separate equating terms of the same order of smallness we can transform the desired dependencies to more convenient view

$$
\begin{aligned}
& b_{i}=\dot{a}_{i}+\sum_{n, m} \dot{a}_{n} a_{m} \gamma_{n m i}^{w}+\sum_{n, m, l} \dot{a}_{n} a_{m} a_{l} \delta_{n m l i}^{w}+\sum_{n, m, l, k} \dot{a}_{n} a_{m} a_{l} a_{k} h_{n m l k i}^{w}, \\
& \vec{q}_{p}=\sum_{j} a_{j} \vec{\beta}_{j p}^{u}+\sum_{j, k} a_{j} a_{k} \gamma_{j} a_{k} \vec{\gamma}_{j k p}^{u}+\sum_{j, k, l} a_{j} a_{k} a_{l} \vec{\delta}_{j k l p}^{u} .
\end{aligned}
$$

The coefficients, which enter the relations $(20),(21)$, represent quadratures of functions $\psi_{i}$ and $\vec{\Omega}_{0}$ computed over the domain $S_{0}$ or of certain expressions, which contains these quadratures. We note that dependencies of the coefficients $b_{i}$ and $\vec{q}_{p}$ on $a_{i}$ and $\dot{a}_{j}$ are obtained in analytical form accurate to values, which guarantee obtaining motion equations accurate to the third order of smallness inclusively for arbitrary number of amplitude parameters. This makes it possible to suppose that accurate to the third order of smallness kinematical boundary condition will hold for arbitrary values of $a_{i}$ and, since we found dependencies of coefficients of decomposition into series of values $\varphi$ and $\vec{\Omega}$ by coefficients of decomposition of $\xi$, then in that way within the framework of accepted restrictions on order of entering values we ascertained approximate dependencies of functions $\varphi$ and $\vec{\Omega}$ on $\xi$. 
Thus, on the basis of use of decompositions of desirable variables by solution of the correspondent eigenvalue linear problem and preliminary satisfying kinematical boundary condition on a free surface of liquid according to stated technique we construct decompositions of desirable variables, which hold identically requirements of flow continuity in the domain $\tau(7)$, kinematical boundary conditions on rigid boundary of the domain $\tau(8)$ and accurate to values of the third order the kinematical boundary condition on a free surface of liquid (9). We note that according to technique of the present paper we can satisfy kinematical boundary condition on a free surface of liquid with arbitrary given accuracy.

Taking into account a fact of satisfying kinematical constraints the Lagrange function (10) now corresponds to a free system, and the system of parameters $a_{i}, \alpha_{i}, \varepsilon_{i}$ is the system of independent variables, which completely characterizes behavior of the mechanical system body - liquid with a free surface.

\section{NONLINEAR DISCRETE MODEL OF DYNAMICS OF COMBINED MOTION OF A BODY WITH LIQUID WITH A FREE SURFACE}

Let us substitute decompositions (12)-(14) into the Lagrange function (10). Since spatial variables are separated and the form of their entering into these decompositions is defined uniquely, we can do integration by spatial variables in all integrals over domains $\tau$, $S$ and $L$. Here determination of integrals over surface $S$ and contour $L$ is elementary, while integration over movable domain should be realize according to the following algorithm. Since the considered domain is cylindrical, then for arbitrary integrand

$$
\int_{\tau} F d \tau=\int_{S_{0}} \int_{-H}^{\xi} F r d r d \theta d z
$$

Determination of integrals with variable upper limit $\xi$ is done on the basis on perturbation methods according to the formula

$$
\int_{a}^{b+\xi} f(z) d z=\int_{a}^{b} f(z) d z+\xi f(b)+\frac{1}{2} \xi^{2} f^{\prime}(b)+\frac{1}{6} \xi^{3} f^{\prime \prime}(b)+\frac{1}{24} \xi^{4} f^{\prime \prime \prime}(b)+\ldots
$$

which is obtained from decomposition of the function $F(\xi)=\int_{-H}^{\xi} f(z) d z$ into Taylor series in a vicinity of $\xi=0$ with assumption of smallness of the value $\xi$.

After integration over spatial variables (we omit intermediate derivations) accurate to values of fourth order we obtain the transformed Lagrange function, which corresponds to discrete model of the considered system

$$
\begin{aligned}
L= & \frac{1}{2} \rho \sum_{i} \dot{a}_{i}^{2} \alpha_{i}^{v}+\frac{1}{2} \rho \sum_{i, j, k} \gamma_{i, j, k}^{v}+\frac{1}{2} \rho \sum_{i, j, k, l} \dot{a}_{i} \dot{a}_{j} a_{k} \delta_{i, j, k, l}^{v} \\
& +\frac{1}{2} \rho \sum_{p, s=1}^{3} \omega_{p} \omega_{s}\left(I_{\text {res }}^{p s}+A_{p s}^{2}+\sum_{i} a_{i} E_{p s i}^{2}+\sum_{i, j} a_{i} a_{j} E_{p s i j}^{2}\right)
\end{aligned}
$$




$$
\begin{aligned}
& +\frac{1}{2} \rho \sum_{p=1}^{3} \omega_{p}\left(\sum_{i} \dot{a}_{i} E_{p i}^{1 *}+\sum_{i, j} \dot{a}_{i} a_{j} E_{p s i j}^{2 *}+\sum_{i, j, k} \dot{a}_{i} a_{j} E_{p i j k}^{3 *}\right) \\
& +\rho \dot{\vec{\varepsilon}} \cdot\left(\sum_{i} \dot{a}_{i} \vec{B}_{i}^{1}+\sum_{i, j} \dot{a}_{i} a_{j} \vec{B}_{i j}^{2}+\sum_{i, j, k} \dot{a}_{i} a_{j} a_{k} B_{i j k}^{3}\right. \\
& \left.+\sum_{i, j, k, l} \dot{a}_{i} a_{j} a_{k} a_{l} \vec{B}_{i j k l}^{4}\right)+\rho \dot{\vec{\varepsilon}} \cdot \sum_{p=1}^{3} \omega_{p}\left(\vec{F}_{p}^{1}+\sum_{i} a_{i} \vec{F}_{p i}^{2}\right. \\
& \left.+\sum_{i, j} a_{i} a_{j} \vec{F}_{p i j}^{3}+\sum_{i, j, k} a_{i} a_{j} a_{k} \vec{F}_{p i j k}^{4}\right)+\frac{1}{2}\left(M_{r}+M_{1}\right)(\dot{\vec{\varepsilon}})^{2} \\
& -\left(M_{r}+M_{1}\right) g \varepsilon_{z}+\rho g\left(\cos \alpha_{1} \sin \alpha_{2} \cos \alpha_{3}-\sin \alpha_{1} \sin \alpha_{3}\right) \\
& \times\left(\sum_{i} a_{i} \alpha_{i}^{c}+H l_{c}\right)-\rho g\left(\sin \alpha_{1} \cos \alpha_{3}+\cos \alpha_{1} \sin \alpha_{2} \sin \alpha_{3}\right) \\
& \times\left(\sum_{i} a_{i} \alpha_{i}^{s}+H l^{s}\right)-\frac{1}{2} \rho g \cos \alpha_{1} \cos \alpha_{2} \sum_{i} a_{i}^{2} N_{i}-\sigma \cos \theta_{1} \\
& \times \sum_{i} a_{i} \lambda_{i}-\sigma S_{0}-\frac{\sigma}{2} \sum_{i} a_{i}^{2} \varkappa_{i}^{2} N_{i}+\frac{\sigma}{8} \sum_{i, j, k, l} a_{i} a_{j} a_{k} a_{l} \delta_{i j k l}^{4} \\
& -\left(M_{r} h_{r}+M_{1} h_{1}\right) g\left(1-\cos \alpha_{1} \cos \alpha_{2}\right)+\overrightarrow{\mathcal{F}} \cdot \vec{\varepsilon}+\vec{M} \cdot \vec{\chi} .
\end{aligned}
$$

We note that in the relation (23) we used the assumption about smallness of angles of inclination of the reservoir, and, therefore, angular velocities too.

The Lagrange equations of the second kind may be obtained from the transformed Lagrange function of a free system.

The system of motion equations in parameters $a_{i}, \varepsilon_{i}, \alpha_{i}$ is supplemented by the generalized dissipative forces, calculated on the basis of results of the book [3]. Here in the equations values $\varepsilon_{i}$ are considered finite, $\alpha_{i}$ and $a_{i}$ are kept accurate to values of the third order inclusively. The system consists of $N+6$ equations of the second order, where $N$ is the number of taken into account normal modes of liquid. This system of equations can be considered as discrete model of the system body - liquid with a free surface.

Significant peculiarity of the constructed algorithm consists in the property that the accepted approach in contrast to other methods makes it possible to obtain nonlinear discrete model of mechanical system of minimal dimension, i.e., its dimension coincides with the number of degrees of freedom of mechanical system, if we understand as the number of degrees of freedom for liquid with a free surface the number of taken into account normal modes. We recall that all these normal modes according to definition do not violate constraints, superimposed on the system, and there independency is provided by linear independence of the system of functions, which describes forms of motion of a free surface of liquid. 
In the generalized form the system motion equations can be represented as

$$
\sum_{n=1}^{N} p_{r n} \ddot{a}_{n}+\sum_{n=N+1}^{N+3} p_{r n} \ddot{\varepsilon}_{n-N}+\sum_{n=N+4}^{N+6} p_{r n} \ddot{\alpha}_{n-N-3}=q_{r}, \quad r=\overline{1, N+6}
$$

where $p_{r n}$ is a quadratic matrix, $q_{r}$ is a vector of $N+6$ dimension, whose elements depend on $t, \dot{a}_{i}, a_{i}, \varepsilon_{i}, \ddot{\varepsilon}_{i}, \alpha_{i}, \dot{\alpha}_{i}$ (explicit dependence of the matrix $p_{r n}$ on $t$ is observed only in the case of liquid outflow from reservoir). The essential peculiarity of the resolving system of ordinary differential equations is reflected in motion equations (24), namely, this system is linear relative to the second derivatives of unknown values. This enables potential of organization of computational process, for which on every step of numerical integration the system (24) is transformed numerically to the Cauchy form and later by means of the Runge-Kutta method integration in time is done. Here on the stage of transformation of the system to normal Cauchy form order of derivatives is reduced by means of introduction of the generalized velocities $\dot{a}_{i}$ as new equitable variables along with amplitude parameters $a_{i}$. However, it is necessary to note that in spite of dimension doubling further it is sufficient to make calculations with matrixes of $N+6$ dimension, but not with $2(N+6)$, which is predetermined by special form of the resolving matrix.

In the general case the system of resolving equations (24) must have infinite dimension. However, there are several physical premises, which enable restriction of consideration of a finite number of normal modes of liquid oscillation. As results of experimental works show in majority of practically significant cases only several first normal modes of oscillations are disturbed considerably. This is caused by the property that higher harmonics in real time are strongly damped due to manifestation of viscosity. Simultaneously these amplitudes are considerably bounded because of specific selective action of capillary forces [8]. Certain theoretical results about fundamental potential of reduction of the resolving system of ordinary differential equation were obtained in publications $[8,9,12,13]$. Results of experimental publications can serve also as strong background for a number of simplifications of mathematical model of the system body - liquid, which are in their turn are not fundamental for reflection of basic properties of mechanical model of the investigated problem.

Let us introduce in the system conventional partition of normal modes of liquid in classes according to degrees of their influence on occurring processes. We include into the first group normal modes of liquid, which predominantly effect on formation of mechanical processes in the system. Their amplitudes are kept in resolving equations accurate to values of the third order of smallness. We relate those normal modes, which first of all introduce qualitative but not quantitative changes to the second group. In most cases these are axisymmetric normal modes, which further predetermine dissymmetry of wave profiles on a free surface of liquid and manifestation of different internal nonlinear constraints. It is expedient to keep their amplitudes accurate to values of the second order of smallness. We include into the third group normal modes, which must provide sufficient steepness of wave profiles on a free surface of liquid, i.e., higher harmonics of spectrum. Such inclusion of higher harmonics is especially significant on investigation of transient modes of motion of bodies with liquid. It is sufficient to study amplitudes of such modes accurate to values of 
the first order of smallness. The described conventional separation of oscillation modes on classes should be done in every definite case on the basis of analysis of physical properties of the system body - liquid and character of excitation of its motion. However, in most cases such separation can be done only by frequencies of normal modes (distribution according to increase of frequencies). For bodies, which have no axis symmetry, it is necessary to include into the number of modes studied in nonlinear statement normal modes for every of main directions of cross-section of the reservoir independently of relation of their frequencies.

The realized on the basis of experimental and some theoretical publication separation of normal modes into classes makes it possible to limit our consideration by finite number of normal nodes (bases function), reduce the number of subject to determination quadratures and simplify considerably computation of multiple sums. The accepted restrictions and assumptions in construction of the model reflect real physical properties of the systems, which are manifested in practical problems of motion of bodies with liquid in the domain of manifestation of nonlinear effects. Neglect or coarse considering of damping makes it possible to consider the present model acceptable first of all for analysis of fast processes and for processes, which occur during several (5-8) periods of oscillations by the first normal mode, when mostly inertial forces are manifested, while influence of dissipation for low-viscous liquids can be either neglected or considered integrally.

\section{MODELLING OF BEHAVIOR OF THE SYSTEM RESERVOIR - LIQUID}

On investigation of inclined motion of reservoir with liquid with a free surface three classes of problems were considered, namely, motion of the system on pendulum support; motion of the system under the presence of outflowing and mode of abrupt switching of thrust of main engine of rocket with liquid fuel. We investigated characteristics of wave motion of liquid, field of pressure, parameters of motion of the reservoir, characteristics of force and moment interaction of liquid with reservoir walls. In all cases we accept the following parameters of the mathematical model, namely, we consider 12 normal modes of liquid oscillation, three first of which were studied according to the theory of the third order, three consequent ones were studied by the theory of second order, and the rest ones were studied within the linear theory.

For investigation of character of influence of rotational motion of reservoir on wave motion of liquid in reservoir we considered four problems about motion of the system body - liquid, which origins after initial perturbation of a free surface of liquid by the first normal mode $a_{1}(0)=0.3 R$. Here we consider the following variants, namely, reservoir performs translation motion $l=\infty$; lengths of pendulum support is $l=5 R ; l=R ; l=0$.

Figure 2 shows law of variation in time of inclination angle of the reservoir (curves in figure are enumerated according to the mentioned variants). We ascertained that oscillations of a free surface of liquid by the first normal mode and angular oscillations of the reservoir occur in antiphase, in the process of oscillations nonlinear character of developed processes is manifested considerably, which results in nonharmonic law of variation in time of rotation angle of the reservoir as early as in vicinity of the third period of the system oscillations. In the case $l_{0}=-0.2 R$ (curve 5 ) the reservoir performs aperiodic motion, which shows unstable character of the system motion for such a way of pendulum 
suspension of the reservoir. We notice non-monotonic character of the dependence of the value of maximum deviation of the reservoir from vertical position on length of pendulum support. This indicates significance of account of resonant properties of component parts of the oscillatory system, namely, liquid with a free surface and reservoir with liquid filling on pendulum support.

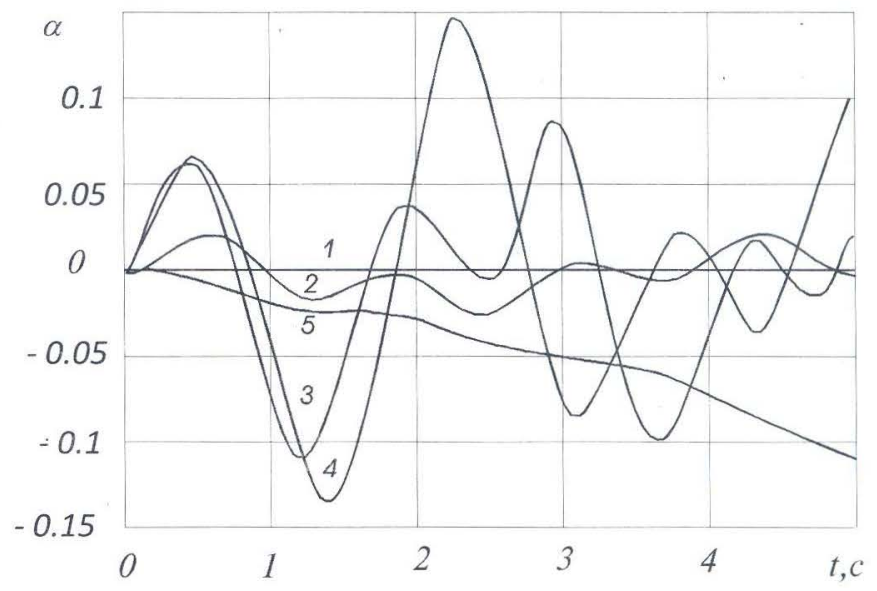

Fig. 2. Variation of inclination angle of the reservoir

On analysis of numerical results we succeeded to discover interesting regularity in character of manifestation of nonlinear constraints in the system depending on length of pendulum support of the reservoir. It was ascertained that for initial stage of the system motion transversal translational motion of the reservoir promote greater manifestation of nonlinear constraints in the system reservoir - liquid with a free surface than inclination motion, for which mainly only the first antisymmetric normal mode of oscillation of liquid with a free surface is disturbed.

On investigation of dynamics of the system reservoir - liquid there is potential of internal resonance between oscillations of a free surface of liquid and oscillations of physical pendulum with internal degrees of freedom, namely, the system reservoir with fixed point - liquid with a free surface. For certain relations between pendulum support length and levels of reservoir filling internal resonance between oscillations of liquid with a free surface and the reservoir with liquid as physical pendulum. We note that the considered internal resonance is observed for small enough levels of filling and small lengths of pendulum support. For example, the first resonance is manifested for $l=0.72 H$. In a vicinity of such lengths of support exchange of energy between quasirigid motion of the system and wave motion of liquid becomes more complicated.

On the basis of the given method we investigated numerically problems about development of transient processes in the system body - liquid for impulse moment disturbance of motion. We consider the case when reservoir with liquid is suspended as physical pendulum with center of support, which coincides with the center of unperturbed free surface 
of liquid $(l=0)$. For numerical examples it was accepted $R=H_{0}=1 \mathrm{~m} ; M_{\mathrm{r}}=0.25 M_{1}$; height of reservoir wall is $H_{\delta}=2 R$; thickness of lateral walls is $0.015 \mathrm{~m}$; thickness of bottom and cover of the reservoir is $0.006 \mathrm{~m}$ (these parameters were used for determination of components of inertia tensor of the reservoir). At start time constant moment acts on the reservoir in the form of tracking transversal force $F=10$ applied at the point, which is for $0.2 R$ lower the suspension center. Force action stops after passing time $\tau=1.5 \mathrm{~s}$.

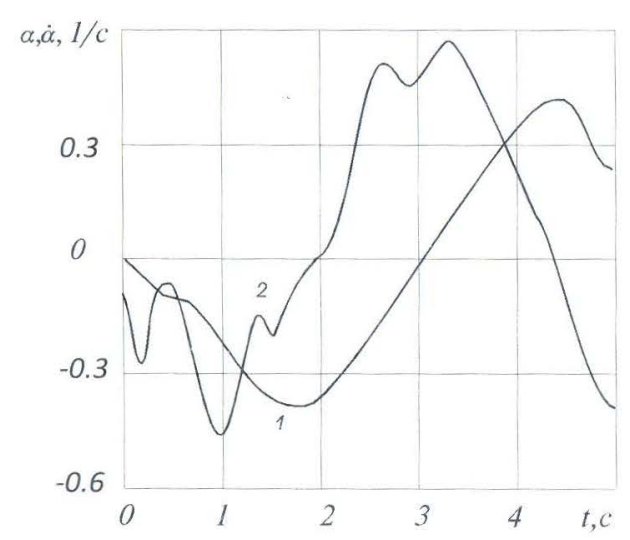

Fig. 3. Variation of turn angle of the reservoir

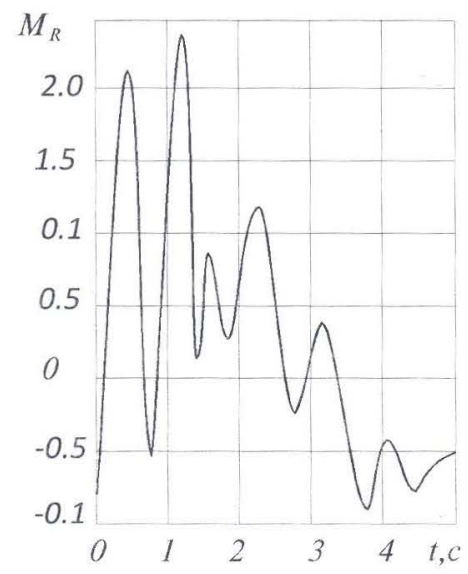

Fig. 4. Variation of main moment of forces

Laws of variation in time of turn angle of the reservoir $\alpha$ relative to the axis $O y$ (curve 1) and angular velocity $\dot{\alpha}$ (curve 2) are shown in Fig. 3. Results of numerical analysis testify the presence of strong interaction of angular motion of a free surface of liquid, which is especially manifested on boost path of system motion. The presence of separate splashes in variation of angular velocity is caused by character of liquid entraining into the process of motion. For the purpose determination of character of force interaction of the reservoir with liquid we consider law of variation in time of the main moment $M_{R}$ of forces relative to suspension center of the reservoir (Figure 4). As it is seen from Fig. 4, in the process of liquid entraining in wave motion the value $M_{R}$ can strongly vary in comparison with rotational motion of liquid coupled with the reservoir. Thus, on time interval before $0.35 \mathrm{~s}$, which approximately corresponds to a quarter of period of oscillations for the first normal mode, $M_{R}$ grows rapidly, after which variation of inclination angle of the reservoir and wave motion of liquid results in liquid motion in opposite direction (wave motion on a free surface of liquid), which, in its turn, results in considerable decrease of of $M_{R}$ and $\dot{\alpha}$ on the interval $0.35-0.36 \mathrm{~s}$. Further, for time instant $0.74 \mathrm{~s}$ (approximately half period of oscillation of the first normal mode) new intense variation of waves on a free surface of liquid, that further predetermines increase of the value $M_{R}$ until time instant $1.2 \mathrm{~s}$. Similar interaction of motion of a free surface of liquid and angular motion the reservoir, caused by variation of direction of motion of wave on a free surface, which corresponds to every of the considered normal mode of liquid, is observed for consequent time instants too. However, on the stage of inertial motion such interaction becomes weaken gradually, which is evidence of installation of concordance, balance of wave motions of liquid and quasirigid angular 
motion of the system on the whole. On active stage, which according to its character represents transient process, system have no time to pass into such concordance.

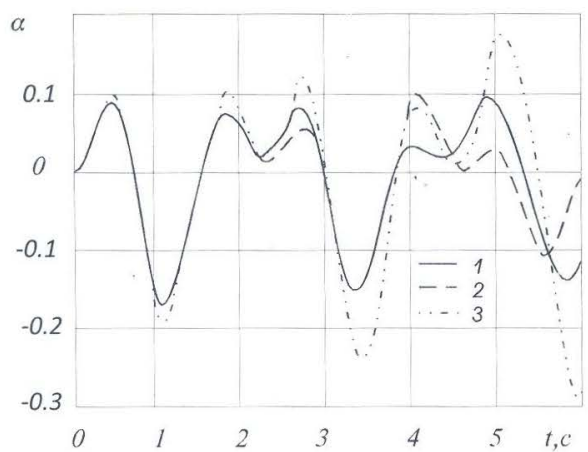

Fig. 5. Variation of turn angle

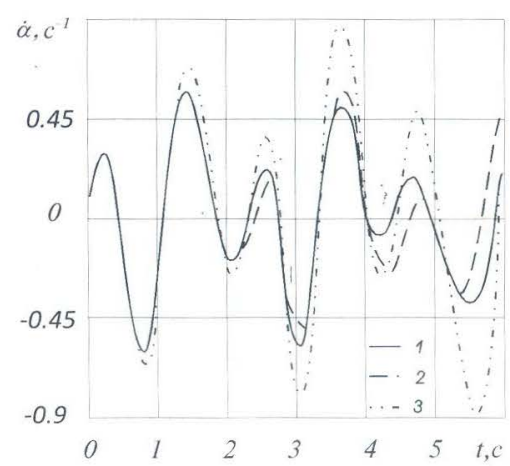

Fig. 6. Variation of angular velocity of the turn angle

Since considerable group of problems is connected with liquid outflow, we considered an example of inclined oscillations of the reservoir on pendulum support under the presence of weak outflow $H=H_{0}+\Delta H t$. Here $l=0.5 R, H_{0}=R, \Delta H=-0.1 \mathrm{~s}^{-1}$, $M_{\mathrm{r}}=0.1 M_{1}, H_{\delta}=1.5 R$, thikness of tank walls was accepted as before. $a_{1}(0)=0.3 R$. Laws of variation in time of turn angle and its angular velocity are represented in Fig. 5 and Fig. 6 correspondingly (dash line describes the case without outflow). Results of modeling show that influence of outflow in the case of inclined oscillations of the reservoir is more significant than in the case of translational motion of the carrying body, however, qualitative regularity of the process remains the same.

The accepted model does not take into account action of reactive forces of the outflowing liquid. For more complete considering of all factors, which accompany liquid outflow from the moving reservoir, we take into account in motion equations reactive forces, which results in appearance of additional terms in motion equations. Let us denote these additions to the right-hand parts of motion equations by $\Delta q_{i}$. Then

$$
\begin{aligned}
& \Delta q_{N+1}=-\frac{M_{1}}{M_{\mathrm{r}}+M_{1}} \frac{\Delta H}{H}\left[\dot{\varepsilon}_{x}-(l+H) \omega_{y}\right] ; \\
& \Delta q_{N+2}=-\frac{M_{1}}{M_{\mathrm{r}}+M_{1}} \frac{\Delta H}{H}\left[\dot{\varepsilon}_{y}+(l+H) \omega_{x}\right] ; \\
& \Delta q_{N+3}=-\frac{M_{1}}{M_{\mathrm{r}}+M_{1}} \frac{\Delta H}{H} \dot{\varepsilon}_{z} ; \\
& \Delta q_{N+3+r}=\frac{M_{1} \Delta H}{\rho H\left(I_{\mathrm{res}}^{p s}+E_{p s}^{2}\right)}\left\{\dot{\varepsilon}_{x} \frac{\partial \omega_{2}}{\partial \dot{\alpha}_{r}}(l+H)-\dot{\varepsilon}_{y} \frac{\partial \omega_{1}}{\partial \dot{\alpha}_{r}}(l+H)\right. \\
& \left.\quad-\left(\frac{\partial \omega_{1}}{\partial \dot{\alpha}_{r}} \omega_{1}+\frac{\partial \omega_{2}}{\partial \dot{\alpha}_{r}} \omega_{2}\right)\left[(l+H)^{2}+\frac{1}{4} R^{2}\left(1+\delta^{2}\right)\right]\right\}, p, s, r=1,2,3,
\end{aligned}
$$


here $\delta$ is ratio of radiuses of internal cylinder to the external one in the case of coaxial cylindrical empty.

As it is seen from figures, account of reactive forces considerably influences variation of parameters of system motion. Here well-known physical experiment, namely, on liquid outflow from oscillation reservoir on pendulum support amplitude of quasirigid oscillations increases, was confirmed.

Let us consider the problem about transient motion of a rocket during main engine thrust shutting off. Subsequent motion of a rocket occurs only under action of thrust of engines of orientation control (steering engines). If we denote thrust of the main engine by $F_{0}$, and thrust of engines of orientation control by $F_{p}$, then during main engine shutting of variation of longitudinal thrust occurs from $F_{0}+F_{p}$ to $F_{p}$ in rather small time interval. For example, in real practice variation of thrust occurs during 1-2 s approximately by linear law for magnitudes $F_{0}=4 g_{0}\left(M_{r}+M_{1}\right), F_{p}=0.1 g_{0}\left(M_{r}+M_{1}\right)$. Here in some cases intense variation of engines thrust, which is named as thrust cutoff, results in abrupt turn of flying object or situations, when energy resources of executing modules of motion stabilization were insufficient for providing prescribed motion. Finally this results in failure or inaccurate realization of object functions. Experimental data makes it possible to state that on thrust cutoff the main mechanism, which lead to violation of desirable orientation of rocket, is formation of antisymmetric splash of liquid, which is accompanied by intense moment response of liquid.

Starting from results of the publication [20], we can suppose that in the considered case with main engine thrust cutoff for variation of overload during 1-2 s we can neglect effects of liquid compressibility. Moreover, it is necessary note that on transition from the initial state $\left(g=4.1 g_{0}\right)$ to terminal one $\left(g=0.1 g_{0}\right)$ effects, connected with capillary forces in tanks with great lateral direction $(2-4 \mathrm{~m})$ will be manifested negligibly.

For problem statement it is important to specify initial state of liquid, which precede thrust cutoff. It is known that in the mode of stationary motion of the system rocket liquid - stabilization module under the presence of thrust in the form of tracking force small antisymmetric oscillations of liquid free surface take place. Further we assume that initial elevation of a free surface by antisymmetric normal mode is approximately equal to $a_{1}(0)=0.1 R$. typical situation, which precedes thrust cutoff, is shown in Fig. 7. Position of mass center of liquid (point $C$ ) creates certain eccentricity of mass in the system. Therefore, it is necessary to expect that tracking force according to its character of action will be equivalent to aggregate action of longitudinal force and certain moment, which depends on eccentricity of mass of the system. In Fig. 7 we show also law of variation of tracking force $F(t)$ in time.

The described situation was modeled on the basis of the developed algorithm. Here we accept the following numerical values of systems parameters, namely, $M_{\mathrm{r}}=0.25 M_{1}$; $H=R ; H_{\delta}=4 R ; R=1.5$; inertia tensor was determined for previously accepted values of walls thicknesses, we consider motion at weightlessness condition and for initial state of liquid we assume that $a_{1}(0)=a_{3}(0)=0.1 R$. Results of numerical modeling of excitation of a free surface of liquid are shown in Fig. 8. Numeration of curves corresponds to position of a free surface at different time instants $1-0 \mathrm{~s} ; 2-0.2 \mathrm{~s} ; 3-0.4 \mathrm{~s} ; 4-0.6 \mathrm{~s}$. 

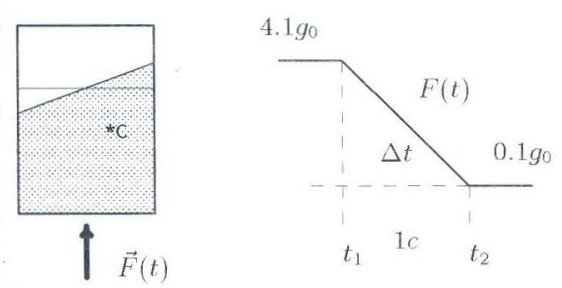

Fig. 7. Variation of tracking force

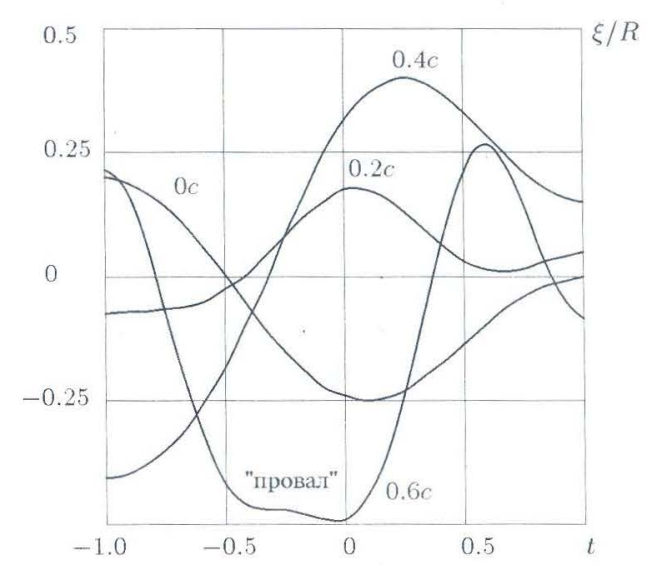

Fig. 8. Excitation of free surface

We can see from figure that tendency of increase of antisymmetric initial perturbations of liquid is manifested and for time instant $0.6 \mathrm{~s}$ maximal splash of liquid forms with free surface of liquid lowering in reservoir center and raise near tank walls. Such phenomenon is called liquid "depression". As it is seen shape of a free surface for $0.6 \mathrm{~s}$ confirms significance of taking into account higher antisymmetric normal modes (we include into the model three first antisymmetric normal modes for every of main directions of motion). For time instant $0.6 \mathrm{~s}$ amplitudes of waves on a free surface reach about $0.5 R$ and further for time instant $t \approx 0.7$ certain effects of loss of stability of numerical procedures are manifested.

For determination of influence of initial perturbations of liquid on future development of in-tank processes for thrust cutoff we considered examples of pure axisymmetric initial perturbation of liquid and development of waves for different variants of antisymmetric and axisymmetric excitations of a free surface.

Results of numerical modeling show that in the axisymmetric case intense development of axisymmetric oscillations occur, caused by the mechanism of development of parametric oscillations, when energy in the system conserves, while potential energy decrease in virtue of variation of longitudinal thrust. Here oscillations of a free surface develop stably for time interval, which exceeds $4 \mathrm{~s}$. At the same time wave processes are developed in different way under the presence of antisymmetric initial perturbations. Here the value of initial antisymmetric elevations of a free surface on the whole influence negligibly on qualitative picture of development of processes during thrust cutoff, while in quantitative sense dependence of development of processes on initial elevations is determinative. We ascertained that on thrust cutoff wave processes on a free surface of liquid develop more intense than for constant thrust because of parametric mechanism. At the same time moment response of liquid for constant trust is greater than in the system with thrust cutoff, that is caused by the property that intensity of decrease of longitudinal overload is higher 
than increase of wave generation for thrust cutoff. It was ascertained also that uncontrollable motion of the system for constant thrust will be unstable. Magnitude of initial antisymmetric elevation of liquid influences slightly on development of processes, while namely fact of presence of antisymmetric elevation install specific motion of liquid, which corresponds to antisymmetric depression and abrupt change of system orientation.

Results of numerical modeling are evidence of the property that mechanism of force oscillations caused by initial mass eccentricity in the system will be determinative, while parametric mechanism is secondary. It is significant to note that on the whole influence of values of initial antisymmetric excitations on development of processes is insignificant, only their presence is decisive. The obtained results qualitatively and in some case quantitatively are agreed with experimental data.

\section{CONCLUSION}

On the basis of use of variational principles of mechanics and analytical elimination of all kinematical constraints we developed the method for construction of finitedimensional model of the system reservoir - liquid with a free surface, which is grounded on principles of modal decomposition. We obtain the system of minimal dimension for arbitrary number of normal modes (the number of unknowns is equal to the number of degrees of freedom).

Peculiarities of development of dynamical processes for angular motion of the carrying body were studied, including the case of pendulum support of the system, which model certain modes of motion for bench-test. On investigation of the initial stage of development of transient process in the system reservoir - liquid it was ascertained that transverse translation excitations of the reservoir provide greater perturbations of nonlinear constraints in the system that inclined motions, for which antisymmetric modes (including higher ones) are excited greater. We investigate the problem about behavior of the structure with liquid for main engine thrust cutoff. It was shown that thrust cutoff results in increase of both symmetric and antisymmetric perturbations of a free surface, including because of increase of higher normal modes. We investigated also variation in time of parameters of liquid motion, reservoir motion and their mutual interaction.

\section{REFERENCES}

[1] K. A. Abgaryan, I. M. Rapoport, Rocket dynamics, Moscow, Mashinostrienie, 1969, p. 378.

[2] Yu. Z. Aleshkov, M.V. Ostrogradskir and his memoirs on wave, Proceedings of Middle-Volga mathematical society 5 (2003) (1) 305-312.

[3] L. G. Boyarshina, P. S. Kovalchuk, Analysis of nonlinear wave motion of liquid in cylindrical vessel, which performs given angular oscillations, Applied Mechanics 26 (1990) (6) 95-101.

[4] L. V. Dokuchaev, V. L. Shetukhin, Stability of non-small oscillation of liquid in a vessel, which performs angular oscillations, Theory of stability and its applications, Novosibirsk, 1979, 100-106.

[5] N. E. Zhukovskiy, On motion of a rigid body, which has empties filled by homogeneous dripping liquid, Collection of works 2 (1931) (1) 136, Moscow-Leningrad, GNTI.

[6] L. V. Kantorovich, V. I. Krylov, Approximate method of high analysis, Moscow - Leningrad, Fizmatgiz, 1962, 708. 
[7] O. S. Limarchenko, Direct method for solving nonlinear problems of dynamics of reservoir carrying liquid, Reports of AN USSR, Ser. A, 1978, No. 11, p. 999-1002.

[8] O. S. Limarchenko, V. V. Yasinskiy, Nonlinear dynamics of structures with liquid, Kiev, National Technical University of Ukraine "Kiev polytechnical Institute", 1997, 338 p.

[9] I. A. Lukovskiy, Introduction to nonlinear dynamics of a rigid body with empties containing liquid, Kiev, Naukova dumka, 1990, p. 295.

[10] A. I. Lourie, Analytical mechanics, Moscow, Fizmatgiz, 1961, p. 824.

[11] S. G. Mikhlin, Variational methods in mathematical physics, Moscow, Nauka, p. 512

[12] N. N. Moiseev, V. V. Roumyantsev, Dynamics of a body with empties, which contain liquid, Moscow, Nauka, 1965, 440 s.

[13] G. S. Narimanov, L. V. Dokuchaev, I. A. Lukovskiy, Nonlinear dynamics of a spacecraft with liquid, Moscow, Mashinostroenie, 1977, p. 208.

[14] M. Faraday, On the forms and states assumed by fluids in contact with vibrating elastic surface, Phil. Trans. of the Royal Society of London 121 (1831) 319-346.

[15] L. A. Ibrahim, Liquid sloshing dynamics, New York: Cambridge Univ. Press, 2005, p. 948.

[16] Lord Rayleigh, On the crispations of fluid resting upon a vibrating support, Philosophical Magazine, 16 (1883) 50-58.

[17] M. La Rocca, G. Sciortino, A generalized variational approach to the sloshing: experimental validation of the model, // 3rd International Conference "Engineering Aero-Hydroelasticity", Prague, August 30-September 3, 1999, P. 256-261.

[18] M. La Rocca, G. Sciortino, M. A. Boniforti, A fully nonlinear model for sloshing in a rotating container, Fluid Dynamics Research (2000) (27) 23-52.

[19] J. Miles, Nonlinear surface waves in closed basins, Journ. Fluid Mech 75 (1976) (3) 419-448.

[20] J. H. Milgram, The motion of a fluid in a cylindrical container with a free surface following vertical impact, J. Fluid Mech 37 (1969) (3) 435-448.

Received February 13, 2009

\section{CHUYỂN ĐÔNNG KÉP CỦA THÙNG CHỨA CHẤT LỎNG VÀ CÁC CHUYỂN ĐÔNNG GÓC CỦA VỎ THÙNG}

Chúng ta xem xét các đặc trưng của các mô hình về chuyển động sóng chất lỏng lý tưởng trong một thùng chứa, thực hiện chuyển động nghiêng. Để mô tả trạng thái của hệ sử dụng công thức biến phân của bài toán dựa trên nguyên tắc biến phân HamiltonOstrogradskiy cơ bản thỏa mãn tất cả các điều kiện biên động học của bài toán. Cách tiếp cận này làm cho hệ có thể làm giảm đáng kể số lượng ẩn số của bài toán và rút gọn hệ chỉ còn các tham số của chuyển động của một bề mặt chất lỏng tự do và các thông số chuyển động của thùng chứa. Mô hình phi tuyến rời rạc đã được xây dựng cho hệ được áp dụng để nghiên cứu chuyển động của thùng chứa chất lỏng trên hệ thống giảm sóc con lắc, có kể đến khối lượng chất lỏng vào và ra và các vấn đề có ý nghĩa lý thuyết và ứng dụng khác. 